\title{
A Roadmap for Disruptive Applications \& Heterogeneous Integration using 2-Dimensional Materials: State-of-the-Art and Technological Challenges
}

Mayank Shrivastava ${ }^{1} \&$ V. Ramgopal Rao ${ }^{2}$

${ }^{1}$ Indian Institute of Science \& ${ }^{2}$ Indian Institute of Technology Delhi; Email: mayank@iisc.ac.in; $\underline{\text { rrao@iitd.ac.in }}$

$\underline{\text { Supplementary Information }}$

Table S1: Electrical and thermal properties of various 2D materials.

\begin{tabular}{|c|c|c|c|c|c|c|}
\hline & Mobility $\left(\mathrm{cm}^{2} / \mathrm{v}-\mathrm{s}\right)$ & $V_{\text {sat }}\left(10^{7} \mathrm{~cm} / \mathrm{sec}\right)$ & $S(\mu \mathrm{V} / \mathbf{K})$ & \multicolumn{2}{|c|}{$\kappa(\mathbf{W} / \mathbf{m K})$} & $\mathrm{ZT}$ \\
\hline & & & & Theoretical & Experimental & \\
\hline Graphene & $\sim 200000$ [S13] & $5.2[\mathrm{~S} 13]$ & $80[\mathrm{~S} 1]$ & $1500-3000[\mathrm{~S} 1]$ & 4840 to $5300[\mathrm{~S} 1]$ & 4@300K [S9] \\
\hline $\begin{array}{l}\mathrm{hBN} \text { (mono \& } \\
\text { few layers) }\end{array}$ & & & & & $100-270[\mathrm{~S} 2]-[\mathrm{S} 4]$ & \\
\hline Phosphorene (BP) & $\sim 1000$ [S13] & & 335 to 510 [S10] & & $\begin{array}{c}30.15 \text { (Zigzag), } 13.65 \\
\text { (Armchair) [S5] }\end{array}$ & $1.1 @ \mathrm{RT}$ [S11] \\
\hline MoS2 & & 0.34 [S13] & $\begin{array}{c}\text { (-)200 to (-) } 300 \\
\text { [S12] }\end{array}$ & & $\begin{array}{c}33.6[\mathrm{~S} 6] \\
34.5 \pm 4[\mathrm{~S} 7]\end{array}$ & \\
\hline $\mathrm{MoSe} 2$ & & & & & $17.6[\mathrm{~S} 6]$ & \\
\hline WS2 & $300-500$ [S13] & & & & $\begin{array}{c}31.8[\mathrm{~S} 6], 32[\mathrm{~S} 13] \\
126 \text { (In-Plane) and } \\
4.7 \text { (Out-of- } \\
\text { Plane) [S8] }\end{array}$ & \\
\hline WSe2 & & & & & $\begin{array}{l}\text { 42(In-Plane) and } \\
3.0 \text { (Out-of- } \\
\text { Plane) [S } 8]\end{array}$ & \\
\hline
\end{tabular}

Table S2: Optical properties of various 2D materials.

\begin{tabular}{|c|c|c|c|c|c|c|c|c|c|c|c|c|c|}
\hline \multirow{2}{*}{ Materials } & \multicolumn{2}{|c|}{ Bandgap (eV) } & \multicolumn{4}{|c|}{ Exiton energy peak (eV) } & \multirow{2}{*}{$\begin{array}{l}\text { Exciton binding } \\
\text { energy }(\mathrm{eV})\end{array}$} & \multirow{2}{*}{$\begin{array}{l}\text { Trion binding } \\
\text { energy (meV) }\end{array}$} & \multirow{2}{*}{$\begin{array}{c}\text { Exciton relaxation } \\
\text { time (ps) }\end{array}$} & \multirow{2}{*}{$\begin{array}{c}\text { Exciton diffusion } \\
\text { length (um) }\end{array}$} & \multicolumn{3}{|c|}{ Raman peaks $\left(\mathrm{cm}^{-1}\right)$} \\
\hline & Monolayer & Bulk & A & B & $\mathrm{A}^{\prime}$ & $\mathrm{B}^{\prime}$ & & & & & & & \\
\hline Graphene & 0 & & & & & & & & & & $\mathrm{D}(1350)[\mathrm{S} 31]$ & $\mathrm{G}(1584)[\mathrm{S} 31]$ & $\mathrm{G}^{\prime}(2700)[\mathrm{S} 31]$ \\
\hline $\mathrm{hBN}$ & $5.955[\mathrm{~S} 24]$ & & & & & & $0.3[\mathrm{~S} 23], 0.13$ [S24] & & & & $\begin{array}{c}\text { E2g (1366) } \\
{[\text { [S31] }}\end{array}$ & & \\
\hline Phosphorene & $2[\mathrm{~S} 22]$ & $0.3[\mathrm{~S} 22]$ & & & & & $0.3[\mathrm{~S} 22]$ & $100[\mathrm{~S} 22]$ & & & $\operatorname{Alg}(360)[S 33]$ & B2g (440) [S33] & A2g (468)[S33] \\
\hline $\mathrm{MoS} 2$ & $\begin{array}{c}2.84[\mathrm{~S} 14], 1.9 \\
{[\mathrm{~S} 26, \mathrm{~S} 27]}\end{array}$ & $1.23[\mathrm{~S} 25], 1.29$ [S26] & $1.88[\mathrm{~S} 14]$ & $2.02[\mathrm{~S} 14]$ & $2.2[\mathrm{~S} 14]$ & $2.32[\mathrm{~S} 14]$ & & 18 [S15] & $300[\mathrm{~S} 30]$ & $1.5-4[\mathrm{~S} 28]$ & \begin{tabular}{|c|} 
E12g(383) \\
{$[$ [S34] }
\end{tabular} & $\operatorname{Alg}(408)[S 34]$ & \\
\hline $\mathrm{MoSe} 2$ & 2.13 [S21],1.57[S27] & 1.09 [S25] & $\begin{array}{l}1.659 \\
{[\mathrm{~S} 16],} \\
1.667 \\
{[\mathrm{~S} 21]}\end{array}$ & $1.885[\mathrm{~S} 21]$ & & & & 30 [S16], 34 [S21] & & & $\mathrm{Alg}(242)$ [S35] & E12g (285)[S35] & \\
\hline WS2 & $2.03[\mathrm{~S} 27]$ & 1.35 [S25] & $\begin{array}{l}2.018 \\
{[\mathrm{~S} 19]} \\
\end{array}$ & & & & & 43 [S19], 28 [S20] & few hundred [S29] & & $\begin{array}{c}\text { E12g (357) } \\
{[\text { S36] }}\end{array}$ & $\operatorname{Alg}(420)[S 36]$ & \\
\hline WSe2 & $\begin{array}{c}2.42[\mathrm{~S} 18], 1.67 \\
{[\mathrm{~S} 27]}\end{array}$ & $1.20[\mathrm{~S} 25]$ & $\begin{array}{c}1.749 \\
{[\mathrm{~S} 17],} \\
1.65[\mathrm{~S} 18]\end{array}$ & $2.05[\mathrm{~S} 18]$ & $2.40[\mathrm{~S} 18]$ & $2.80[\mathrm{~S} 18]$ & & 30 [S17] & & & $\begin{array}{c}250 \text { (both peaks } \\
\text { ) [S37] }\end{array}$ & & \\
\hline
\end{tabular}


References

S1. A. A. Balandin et al., "Superior thermal conductivity of single-layer graphene," Nano Lett., vol. 8, no. 3, pp. 902-907, 2008, doi: 10.1021/n10731872.

S2. H. Zhou et al. "High thermal conductivity of suspended few-layer hexagonal boron nitride sheets," Nano Res., vol. 7, no. 8, pp. 1232-1240, 2014, doi: 10.1007/s12274-014-0486-Z.

S3. M. T. Alam, M. S. Bresnehan, J. A. Robinson, and M. A. Haque, "Thermal conductivity of ultra-thin chemical vapor deposited hexagonal boron nitride films," Appl. Phys. Lett., vol. 104, no. 1, pp. 1-6, 2014, doi: 10.1063/1.4861468.

S4. I. Jo et al., "Thermal conductivity and phonon transport in suspended few-layer hexagonal boron nitride," Nano Lett., vol. 13, no. 2, pp. 550-554, 2013, doi: 10.1021/n1304060g.

S5. G. Qin, Q.-B. Yan, Z. Qin, S.-Y. Yue, M. Hu, and G. Su, “Anisotropic intrinsic lattice thermal conductivity of phosphorene from first principles,” Phys. Chem. Chem. Phys., vol. 17, no. 7, pp. 4854-4858, 2015, doi: $10.1039 / \mathrm{c} 4 \mathrm{cp} 04858 \mathrm{j}$

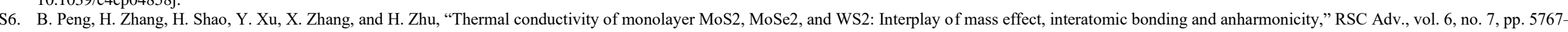
5773, 2016, doi: 10.1039/c5ra19747c.

S7. R. Yan et al., "Thermal conductivity of monolayer molybdenum disulfide obtained from temperature-dependent Raman spectroscopy," ACS Nano, vol. 8, no. 1, pp. 986-993, 2014, doi: 10.1021/nn405826k.

S8. D. O. Lindroth and P. Erhart, "Thermal transport in van der Waals solids from first-principles calculations," Phys. Rev. B, vol. 94, no. 11, pp. 1-11, 2016, doi: 10.1103/PhysRevB.94.115205.

S9. H. Sevinçli and G. Cuniberti, "Enhanced thermoelectric figure of merit in edge-disordered zigzag graphene nanoribbons," Phys. Rev. B - Condens. Matter Mater. Phys., vol. 81, no. 11, pp. 1-4, 2010, doi: 10.1103/PhysRevB.81.113401.

S10. Y. Saito, T. Iizuka, T. Koretsune, R. Arita, S. Shimizu, and Y. Iwasa, "Gate-Tuned Thermoelectric Power in Black Phosphorus," Nano Lett., vol. 16, no. 8, pp. 4819-4824, 2016, doi: 10.1021/acs.nanolett.6b00999.

S11. J. Zhang et al., "High thermoelectric performance can be achieved in black phosphorus," J. Mater. Chem. C, vol. 4, no. 5, pp. 991-998, 2016, doi: 10.1039/c5tc03238e.

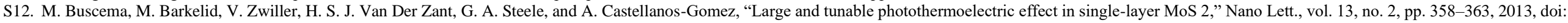
$10.1021 / \mathrm{nl} 303321 \mathrm{~g}$.

S13. F. Schwierz et. al. Nanoscale, 2015, 7,8261

S14. Qiu, D.Y., Felipe, H. and Louie, S.G., 2013. Optical spectrum of MoS 2: many-body effects and diversity of exciton states. Physical review letters, 111(21), p.216805.

S15. Mak, K.F., He, K., Lee, C., Lee, G.H., Hone, J., Heinz, T.F. and Shan, J., 2013. Tightly bound trions in monolayer MoS 2. Nature materials, 12(3), pp.207-211.

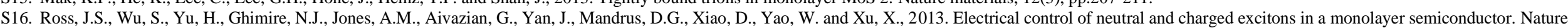
communications, 4(1), pp.1-6.

S17. Jones, A.M., Yu, H., Ghimire, N.J., Wu, S., Aivazian, G., Ross, J.S., Zhao, B., Yan, J., Mandrus, D.G., Xiao, D. and Yao, W., 2013. Optical generation of excitonic valley coherence in monolayer WSe 2. Nature nanotechnology, 8(9), pp.634-638.

S18. Del Corro, E., Terrones, H., Elias, A., Fantini, C., Feng, S., Nguyen, M.A., Mallouk, T.E., Terrones, M. and Pimenta, M.A., 2014. Excited excitonic states in 1L, 2L, 3L, and bulk WSe2 observed by resonant Raman spectroscopy. Acs Nano, 8(9), pp.9629-9635.

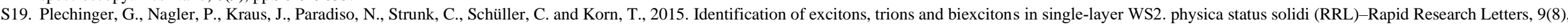
pp.457-461.

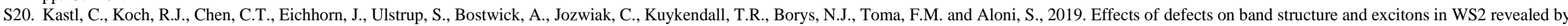
nanoscale photoemission spectroscopy. ACS nano, 13(2), pp.1284-1291.

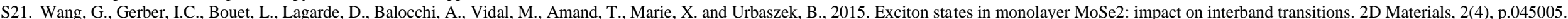

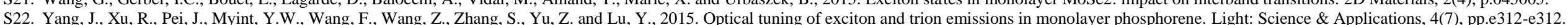

S23. Plaud, A., Schué, L., Watanabe, K., Taniguchi, T., Fossard, F., Ducastelle, F., Loiseau, A. and Barjon, J., 2019. Exciton-exciton annihilation in hBN. Applied Physics Letters, 114(23), p.232103.

S24. Causabois, G., Valvin, P. and Gil, B., 2016. Hexagonal boron nitride is an indirect bandgap semiconductor. Nature photonics, 10(4), pp.262-266

S24. Cassabois, G., Valvin, P. and Gil, B., 2016. Hexagonal boron

S25. K. K. Kam, B. A. Parkinson, J. Phys. Chem. 1982, 86, 463.
S26. Mak, K.F., Lee, C., Hone, J., Shan, J. and Heinz, T.F., 2010. Atomically thin MoS 2: a new direct-gap semiconductor. Physical review letters, $105(13)$, p.136805.

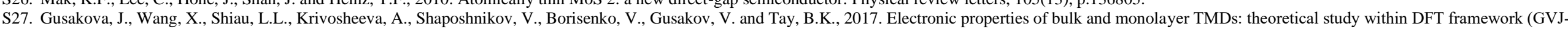
2e method). physica status solidi (a), 214(12), p.1700218.

S28. Uddin, S.Z., Kim, H., Lorenzon, M., Yeh, M., Lien, D.H., Barnard, E.S., Htoon, H., Weber-Bargioni, A. and Javey, A., 2020. Neutral exciton diffusion in monolayer MoS2. ACS nano, 14(10), pp.13433-13440.

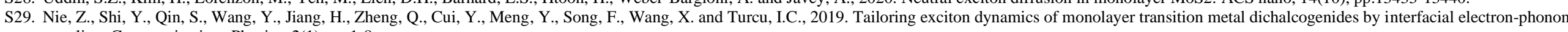
coupling. Communications Physics, 2(1), pp.1-8.

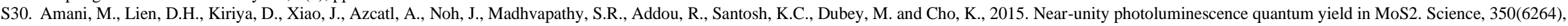
pp.1065-1068.

S31. Ferrari, Andrea C., and Denis M. Basko. "Raman spectroscopy as a versatile tool for studying the properties of graphene." Nature nanotechnology 8, no. 4 (2013): $235-246$.

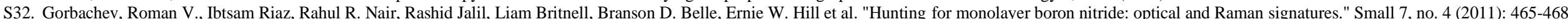

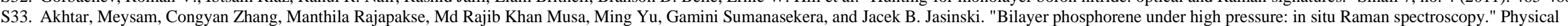
Chemistry Chemical Physics 21, no. 14 (2019): 7298-7304

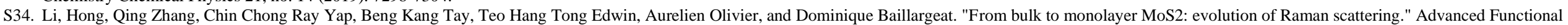
Materials 22, no. 7 (2012): 1385-1390.

S35. Soubelet, P., Axel Emerico Bruchhausen, Alejandro Fainstein, K. Nogajewski, and C. Faugeras. "Resonance effects in the Raman scattering of monolayer and few-layer MoSe 2." Physical Review B 93, no. 15 (2016): 155407

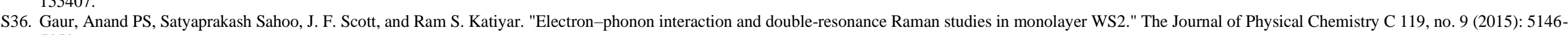
5151

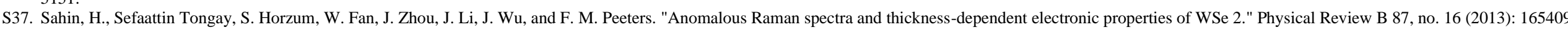

